Stathis Filippas · Achilles Tertikas · Jesper Tidblom

\title{
On the structure of Hardy-Sobolev-Maz'ya inequalities
}

Received October 11, 2007 and in revised form February 6, 2008

Abstract. We establish new improvements of the optimal Hardy inequality in the half-space. We first add all possible linear combinations of Hardy type terms, thus revealing the structure of this type of inequalities and obtaining best constants. We then add the critical Sobolev term and obtain necessary and sufficient conditions for the validity of Hardy-Sobolev-Maz'ya type inequalities.

\section{Introduction}

One version of the Hardy inequality states that for convex domains $\Omega \subset \mathbb{R}^{n}$ the following estimate holds:

$$
\int_{\Omega}|\nabla u|^{2} d x \geq \frac{1}{4} \int_{\Omega} \frac{|u|^{2}}{d(x)^{2}} d x, \quad u \in C_{0}^{\infty}(\Omega),
$$

where $d(x)=\operatorname{dist}(x, \partial \Omega)$ and the constant $1 / 4$ is best possible. This result has been improved and generalized in many different ways: see for example [1], [2], [4]-[9], [12], [13].

One pioneering result due to Brezis and Marcus [4] is the following improved Hardy inequality:

$$
\int_{\Omega}|\nabla u|^{2} d x \geq \frac{1}{4} \int_{\Omega} \frac{|u|^{2}}{d(x)^{2}} d x+C_{2}(\Omega) \int_{\Omega}|u|^{2} d x, \quad u \in C_{0}^{\infty}(\Omega),
$$

valid for any convex domain $\Omega \subset \mathbb{R}^{n}$. This estimate has recently been extended in [7]:

$$
\int_{\Omega}|\nabla u|^{2} d x \geq \frac{1}{4} \int_{\Omega} \frac{|u|^{2}}{d(x)^{2}} d x+C_{q}(\Omega)\left(\int_{\Omega}|u|^{q} d x\right)^{2 / q}, \quad u \in C_{0}^{\infty}(\Omega) .
$$

S. Filippas: Department of Applied Mathematics, University of Crete, 71409 Heraklion, Greece, and Institute of Applied and Computational Mathematics, FORTH, 71110 Heraklion, Greece; e-mail: filippas@tem.uoc.gr

A. Tertikas: Department of Mathematics, University of Crete, 71409 Heraklion, Greece, and Institute of Applied and Computational Mathematics, FORTH, 71110 Heraklion, Greece; e-mail: tertikas@math.uoc.gr

J. Tidblom: The Erwin Schrödinger Institute (ESI), Boltzmanngasse 9, A-1090 Vienna, Austria; e-mail: Jesper.Tidblom@esi.ac.at 
Moreover, it is shown in [7] that there exist constants $c_{1}$ and $c_{2}$ only depending on $q$ and the dimension $n$ of $\Omega$ such that the best constant $C_{q}(\Omega)$ satisfies

$$
c_{1} D^{n-2-2 n / q} \geq C_{q}(\Omega) \geq c_{2} D^{n-2-2 n / q},
$$

where $D=\sup _{x \in \Omega} d(x)<\infty$ and $2 \leq q<2 n /(n-2)$. We note that the critical Sobolev exponent $q=2^{*}:=2 n /(n-2)$ is not included in the above theorem. For results in the critical case we refer to [8].

Let $S_{n}=\pi n(n-2)(\Gamma(n / 2) / \Gamma(n))^{2 / n}, n \geq 3$, denote the best constant in the Sobolev inequality

$$
\int_{\Omega}|\nabla u|^{2} d x \geq S_{n}\left(\int_{\Omega}|u|^{2^{*}} d x\right)^{2 / 2^{*}}, \quad u \in C_{0}^{\infty}(\Omega) .
$$

The first inequality that combines both the critical Sobolev exponent term and the Hardy term, the latter with best constant, is due to Maz'ya [10], and is the following HardySobolev-Maz'ya inequality:

$$
\int_{\mathbb{R}_{+}^{n}}|\nabla u|^{2} d x \geq \frac{1}{4} \int_{\mathbb{R}_{+}^{n}} \frac{|u|^{2}}{x_{1}^{2}} d x+C_{n}\left(\int_{\mathbb{R}_{+}^{n}}|u|^{2^{*}} d x\right)^{2 / 2^{*}}, \quad u \in C_{0}^{\infty}\left(\mathbb{R}_{+}^{n}\right),
$$

where $\mathbb{R}_{+}^{n}=\left\{\left(x_{1}, \ldots, x_{n}\right): x_{1}>0\right\}$ denotes the upper half-space, $C_{n}$ is a positive constant and $2^{*}=2 n /(n-2), n \geq 3$. Recently, it was shown in [3] that in the 3 dimensional case, the best constant $C_{3}$ coincides with the best Sobolev constant $S_{3}$ ! On the other hand, when $n \geq 4$ one has $C_{n}<S_{n}$ (see [11]).

We next mention an improvement of Hardy's inequality that involves two distance functions:

$$
\int_{\mathbb{R}_{+}^{n}}|\nabla u|^{2} d x \geq \frac{1}{4} \int_{\mathbb{R}_{+}^{n}} \frac{|u|^{2}}{x_{1}^{2}} d x+C(\tau) \int_{\mathbb{R}_{+}^{n}} \frac{|u|^{2}}{x_{1}^{2-\tau}\left(x_{1}^{2}+x_{2}^{2}\right)^{\tau / 2}} d x, \quad u \in C_{0}^{\infty}\left(\mathbb{R}_{+}^{n}\right),
$$

where $0<\tau \leq 1$. This is a special case of a more general inequality proved in [13].

In this work we study improvements of Hardy's inequality that involve various distance functions. Working in the upper half-space $\mathbb{R}_{+}^{n}$, we obtain Hardy type inequalities that involve constant multiples of the inverse square of the distance to linear submanifolds of different codimensions of the boundary $\partial \mathbb{R}_{+}^{n}$. Actually, we are able to give a complete description of the structure of this kind of improved Hardy inequalities. In particular, we have a lot of freedom in choosing these constants and we will show that all our configurations of constants are, in a natural sense, optimal. More precisely, our first result reads:

Theorem A (Improved Hardy inequality).

(i) Let $\alpha_{1}, \ldots, \alpha_{n}$ be arbitrary real numbers and

$$
\beta_{1}=-\alpha_{1}^{2}+1 / 4, \quad \beta_{m}=-\alpha_{m}^{2}+\left(\alpha_{m-1}-1 / 2\right)^{2}, \quad m=2, \ldots, n .
$$

Then for any $u \in C_{0}^{\infty}\left(\mathbb{R}_{+}^{n}\right)$,

$$
\int_{\mathbb{R}_{+}^{n}}|\nabla u|^{2} d x \geq \int_{\mathbb{R}_{+}^{n}}\left(\frac{\beta_{1}}{x_{1}^{2}}+\frac{\beta_{2}}{x_{1}^{2}+x_{2}^{2}}+\cdots+\frac{\beta_{n}}{x_{1}^{2}+\cdots+x_{n}^{2}}\right) u^{2} d x .
$$


(ii) Suppose that for some real numbers $\beta_{1}, \ldots, \beta_{n}$ the inequality

$$
\int_{\mathbb{R}_{+}^{n}}|\nabla u|^{2} d x \geq \int_{\mathbb{R}_{+}^{n}}\left(\frac{\beta_{1}}{x_{1}^{2}}+\frac{\beta_{2}}{x_{1}^{2}+x_{2}^{2}}+\cdots+\frac{\beta_{n}}{x_{1}^{2}+\cdots+x_{n}^{2}}\right) u^{2} d x
$$

holds for any $u \in C_{0}^{\infty}\left(\mathbb{R}_{+}^{n}\right)$. Then there exist nonpositive constants $\alpha_{1}, \ldots, \alpha_{n}$ such that

$$
\beta_{1}=-\alpha_{1}^{2}+1 / 4, \quad \beta_{m}=-\alpha_{m}^{2}+\left(\alpha_{m-1}-1 / 2\right)^{2}, \quad m=2, \ldots, n .
$$

We next investigate the possibility of adding Sobolev type remainder terms. It turns out that almost every choice of the constants in Theorem A allows one to add a positive Sobolev term as well. The details are in our second main theorem.

Theorem B (Improved Hardy-Sobolev-Maz'ya inequality). Let $\alpha_{1}, \ldots, \alpha_{n}$ be arbitrary nonpositive real numbers and

$$
\beta_{1}=-\alpha_{1}^{2}+1 / 4, \quad \beta_{m}=-\alpha_{m}^{2}+\left(\alpha_{m-1}-1 / 2\right)^{2}, \quad m=2, \ldots, n .
$$

If $\alpha_{n}<0$ then there exists a positive constant $C$ such that for any $u \in C_{0}^{\infty}\left(\mathbb{R}_{+}^{n}\right)$,

$$
\begin{aligned}
\int_{\mathbb{R}_{+}^{n}}|\nabla u|^{2} d x \geq & \int_{\mathbb{R}_{+}^{n}}\left(\frac{\beta_{1}}{x_{1}^{2}}+\frac{\beta_{2}}{x_{1}^{2}+x_{2}^{2}}+\cdots+\frac{\beta_{n}}{x_{1}^{2}+\cdots+x_{n}^{2}}\right) u^{2} d x \\
& +C\left(\int_{\mathbb{R}_{+}^{n}}|u|^{2^{*}} d x\right)^{2 / 2^{*}} .
\end{aligned}
$$

If $\alpha_{n}=0$ then there is no positive constant $C$ such that 1.4 holds.

It is interesting to note that the Sobolev term vanishes precisely when the constant $\beta_{n}$, in front of the Hardy-type term containing the point singularity, is chosen optimal. It is a bit curious that the size of the other constants, $\beta_{1}, \ldots, \beta_{n-1}$, does not matter at all for this question. Only the relative size of $\beta_{n}$ compared to the other constants matters.

Our results depend heavily on the Gagliardo-Nirenberg-Sobolev inequality and also on an interesting relation between the existence of an $L^{1}$ Hardy inequality and the possibility of adding a Sobolev type remainder term to the corresponding $L^{2}$ inequality. The precise result reads:

Theorem C. Let $\Omega \subset \mathbb{R}^{n}, n \geq 3$, be a smooth domain. Assume that $\phi \in C^{2}(\Omega), \phi>0$, and the following weighted $L^{1}$ inequality holds:

$$
\int_{\Omega} \phi^{\frac{2(n-1)}{n-2}}|\nabla v| d x \geq C \int_{\Omega} \phi^{\frac{n}{n-2}}|\nabla \phi||v| d x, \quad v \in C_{0}^{\infty}(\Omega) .
$$

Then there exists $c>0$ such that

$$
\int_{\Omega}|\nabla u|^{2} d x \geq-\int_{\Omega} \frac{\Delta \phi}{\phi}|u|^{2} d x+c\left(\int_{\Omega}|u|^{2^{*}} d x\right)^{2 / 2^{*}}, \quad u \in C_{0}^{\infty}(\Omega) .
$$


The regularity assumptions on $\phi$ can be weakened, but for our purposes it is enough to restrict ourselves to $\phi \in C^{2}(\Omega)$. We note that under the sole assumption $\phi>0$ and $\phi \in C^{2}(\Omega)$ the inequality

$$
\int_{\Omega}|\nabla u|^{2} d x \geq-\int_{\Omega} \frac{\Delta \phi}{\phi}|u|^{2} d x, \quad u \in C_{0}^{\infty}(\Omega),
$$

is always true; see Lemma 2.1 It is the validity of $(1.5)$ that makes possible the addition of the Sobolev term in (1.7). An easy example where both 1.5 and 1.6 fail is the case where $\phi$ is taken to be the first Dirichlet eigenfunction of the Laplacian of $\Omega$, for $\Omega$ bounded.

Our methods are not restricted to the case $\Omega=\mathbb{R}_{+}^{n}$. In the last section of the paper we give an example of how to apply the method to get some results for the quarter-space. Moreover, as one can easily check, our results remain valid even for complex-valued functions.

The paper is organized as follows. In Section 2 we give the proof of Theorem A. In Section 3 we prove Theorems B and C. Finally, in the last section we obtain some results for the quarter-space.

\section{Improved Hardy inequalities in the half-space}

The half-space $\mathbb{R}_{+}^{n}$ has some nice features that are not present for an arbitrary convex domain. The fact that the boundary has zero curvature is very useful when one tries to prove certain sorts of inequalities, as we shall see below.

We start with a general auxiliary lemma.

Lemma 2.1. (i) Let $\mathbf{F} \in C^{1}(\Omega)$. Then

$$
\int_{\Omega}|\nabla u|^{2} d x=\int_{\Omega}\left(\operatorname{div} \mathbf{F}-|\mathbf{F}|^{2}\right)|u|^{2} d x+\int_{\Omega}|\nabla u+\mathbf{F} u|^{2} d x, \quad u \in C_{0}^{\infty}(\Omega) .
$$

(ii) Let $\phi>0, \phi \in C^{2}(\Omega)$ and $u=\phi v$. Then

$$
\int_{\Omega}|\nabla u|^{2} d x=-\int_{\Omega} \frac{\Delta \phi}{\phi} u^{2} d x+\int_{\Omega} \phi^{2}|\nabla v|^{2} d x, \quad u \in C_{0}^{\infty}(\Omega) .
$$

Proof. By expanding the square we have

$$
\int_{\Omega}|\nabla u+\mathbf{F} u|^{2} d x=\int_{\Omega}|\nabla u|^{2} d x+\int_{\Omega}|\mathbf{F}|^{2} u^{2} d x+\int_{\Omega} \mathbf{F} \cdot \nabla u^{2} d x .
$$

Identity 2.1 now follows by integrating by parts the last term.

To prove 2.2 we apply 2.1] to $\mathbf{F}=-\nabla \phi / \phi$. Elementary calculations now yield the result. 
We especially want to study inequalities of the type

$$
\begin{aligned}
& \int_{\mathbb{R}_{+}^{n}}|\nabla u|^{2} d x \\
& \geq \int_{\mathbb{R}_{+}^{n}}\left(\frac{\beta_{1}}{x_{1}^{2}}+\frac{\beta_{2}}{x_{1}^{2}+x_{2}^{2}}+\cdots+\frac{\beta_{n}}{x_{1}^{2}+x_{2}^{2}+\ldots+x_{n}^{2}}\right)|u|^{2} d x, \quad u \in C_{0}^{\infty}\left(\mathbb{R}_{+}^{n}\right),
\end{aligned}
$$

where $\beta=\left(\beta_{1}, \ldots, \beta_{n}\right)$ is a vector of nonnegative constants. The case when $\beta_{1}=1 / 4$ is especially interesting since it corresponds to the term in the standard Hardy inequality. So every legitimate choice of $\beta$ with $\beta_{1}=1 / 4$ corresponds to an improved Hardy inequality. Let us introduce some notation. Let

$$
\mathbf{X}_{k}:=\left(x_{1}, \ldots, x_{k}, 0, \ldots, 0\right) \text { so that }\left|\mathbf{X}_{k}\right|^{2}=x_{1}^{2}+\cdots+x_{k}^{2} .
$$

We now give the proof of the first part of Theorem A:

Proof of Theorem $A(i)$. Let $\gamma_{1}, \ldots, \gamma_{n}$ be arbitrary real numbers and set

$$
\phi:=\left|\mathbf{X}_{1}\right|^{-\gamma_{1}} \ldots\left|\mathbf{X}_{n}\right|^{-\gamma_{n}}, \quad \mathbf{F}:=-\frac{\nabla \phi}{\phi} .
$$

An easy calculation shows that

$$
\mathbf{F}=\sum_{m=1}^{n} \gamma_{m} \frac{\mathbf{X}_{m}}{\left|\mathbf{X}_{m}\right|^{2}}
$$

With this choice of $\mathbf{F}$, we get

$$
\operatorname{div} \mathbf{F}=\sum_{m=1}^{n} \gamma_{m} \frac{m-2}{\left|\mathbf{X}_{m}\right|^{2}}
$$

and

$$
|\mathbf{F}|^{2}=\sum_{m=1}^{n} \frac{\gamma_{m}^{2}}{\left|\mathbf{X}_{m}\right|^{2}}+2 \sum_{m=1}^{n} \sum_{j=1}^{m-1} \gamma_{m} \gamma_{j} \frac{\mathbf{X}_{m}}{\left|\mathbf{X}_{m}\right|^{2}} \frac{\mathbf{X}_{j}}{\left|\mathbf{X}_{j}\right|^{2}}=\sum_{m=1}^{n} \frac{\gamma_{m}^{2}}{\left|\mathbf{X}_{m}\right|^{2}}+2 \sum_{m=1}^{n} \sum_{j=1}^{m-1} \frac{\gamma_{m} \gamma_{j}}{\left|\mathbf{X}_{j}\right|^{2}}
$$

We then get

$$
-\frac{\Delta \phi}{\phi}=\operatorname{div} \mathbf{F}-|\mathbf{F}|^{2}=\sum_{m=1}^{n} \frac{\beta_{m}}{\left|\mathbf{X}_{m}\right|^{2}}
$$

where

$$
\beta_{1}=-\gamma_{1}\left(\gamma_{1}+1\right), \quad \beta_{m}=-\gamma_{m}\left(2-m+\gamma_{m}+2 \sum_{j=1}^{m-1} \gamma_{j}\right), \quad m=2, \ldots, n .
$$

We next set

$$
\gamma_{1}=\alpha_{1}-1 / 2, \quad \gamma_{m}=\alpha_{m}-\alpha_{m-1}+1 / 2, \quad m=2, \ldots, n .
$$

With this choice of $\gamma$ 's the $\beta$ 's are given as in the statement of the theorem. 
As a consequence of Lemma 2.1 we have

$$
\int_{\mathbb{R}_{+}^{n}}|\nabla u|^{2} d x \geq \int_{\mathbb{R}_{+}^{n}}\left(\operatorname{div} \mathbf{F}-|\mathbf{F}|^{2}\right) u^{2} d x .
$$

The result then follows from 2.3 and 2.4).

Remark. It is easy to check that for any choice of $n$ real numbers $\alpha_{1}, \ldots, \alpha_{n}$, we can find $n$ nonpositive real numbers $\alpha_{1}^{\prime}, \ldots, \alpha_{n}^{\prime}$ that give the same constants $\beta_{1}, \ldots, \beta_{n}$. Consequently, without loss of generality, we may assume that $\alpha_{1}, \ldots, \alpha_{n}$ are nonpositive.

In the above theorem we have a lot of freedom. We can choose the $\gamma$ 's in many different ways, each choice giving a different inequality. We may, for instance, first maximize $\beta_{1}$ and then $\beta_{2}$ and so on. More generally, we might try to make the first $m-1 \beta_{m}$ 's equal to zero and then maximize the $\beta_{m}$ 's in increasing order.

In fact, we have the following corollary:

Corollary 2.2. Let $k=1, \ldots, n$. Then

$$
\begin{aligned}
\int_{\mathbb{R}_{+}^{n}}|\nabla u|^{2} d x \geq & \int_{\mathbb{R}_{+}^{n}}\left(\frac{k^{2}}{4} \frac{1}{x_{1}^{2}+\cdots+x_{k}^{2}}+\frac{1}{4} \frac{1}{x_{1}^{2}+\cdots+x_{k+1}^{2}}+\cdots\right. \\
& \left.+\frac{1}{4} \frac{1}{x_{1}^{2}+\cdots+x_{n}^{2}}\right) u^{2} d x, \quad u \in C_{0}^{\infty}\left(\mathbb{R}_{+}^{n}\right) .
\end{aligned}
$$

Proof. In the case $k=1$ we choose $\alpha_{1}=\cdots=\alpha_{n}=0$. In this case all $\beta_{k}$ 's are equal to $1 / 4$.

In the general case $k>1$ we choose $\alpha_{m}=-m / 2$ when $m=1, \ldots, k-1$, and $\alpha_{m}=0$ when $m=k, \ldots, n$.

We next prove the second part of Theorem A:

Proof of Theorem A(ii). We will first prove that $\beta_{1} \leq 1 / 4$, therefore $\beta_{1}=-\alpha_{1}^{2}+1 / 4$ for suitable $\alpha_{1} \leq 0$. Then, for this $\beta_{1}$, we will prove that $\beta_{2} \leq\left(\alpha_{1}-1 / 2\right)^{2}$, and therefore $\beta_{2}=-\alpha_{2}^{2}+\left(\alpha_{1}-1 / 2\right)^{2}$ for suitable $\alpha_{2} \leq 0$, and so on.

Step 1. Let us first prove the estimate for $\beta_{1}$. To this end we set

$$
Q_{1}[u]:=\frac{\int_{\mathbb{R}_{+}^{n}}|\nabla u|^{2} d x-\sum_{i=2}^{n} \beta_{i} \int_{\mathbb{R}_{+}^{n}} \frac{u^{2}}{\left(x_{1}^{2}+\cdots+x_{i}^{2}\right)} d x}{\int_{\mathbb{R}_{+}^{n}} \frac{u^{2}}{x_{1}^{2}} d x} .
$$

We clearly have $\beta_{1} \leq \inf _{u \in C_{0}^{\infty}\left(\mathbb{R}_{+}^{n}\right)} Q_{1}[u]$. We will show that

$$
\inf _{u \in C_{0}^{\infty}\left(\mathbb{R}_{+}^{n}\right)} Q_{1}[u] \leq 1 / 4,
$$

whence $\beta_{1} \leq 1 / 4$. 
At this point we introduce a family of cutoff functions for later use. For $j=1, \ldots, n$ and $k_{j}>0$ we set

$$
\phi_{j}(t)= \begin{cases}0, & t<1 / k_{j}^{2} \\ 1+\frac{\ln k_{j} t}{\ln k_{j}}, & 1 / k_{j}^{2} \leq t<1 / k_{j} \\ 1, & t \geq 1 / k_{j}\end{cases}
$$

and

$$
h_{k_{j}}(x):=\phi_{j}\left(r_{j}\right) \quad \text { where } \quad r_{j}:=\left|\mathbf{X}_{j}\right|=\left(x_{1}^{2}+\cdots+x_{j}^{2}\right)^{1 / 2} .
$$

Note that

$$
\left|\nabla h_{k_{j}}(x)\right|^{2}= \begin{cases}\frac{1}{\ln ^{2} k_{j}} \frac{1}{r_{j}^{2}}, & 1 / k_{j}^{2} \leq r_{j} \leq 1 / k_{j}, \\ 0, & \text { otherwise. }\end{cases}
$$

We also denote by $\phi(x)$ a radially symmetric $C_{0}^{\infty}\left(\mathbb{R}^{n}\right)$ function such that $\phi=1$ for $|x|<1 / 2$ and $\phi=0$ for $|x|>1$.

To prove 2.6 we consider the family of functions

$$
u_{k_{1}}(x)=x_{1}^{1 / 2} h_{k_{1}}(x) \phi(x) .
$$

We will show that as $k_{1} \rightarrow \infty$,

$$
\frac{\int_{\mathbb{R}_{+}^{n}}\left|\nabla u_{k_{1}}\right|^{2} d x-\sum_{i=2}^{n} \beta_{i} \int_{\mathbb{R}_{+}^{n}} \frac{u_{k_{1}}^{2}}{\left(x_{1}^{2}+\cdots+x_{i}^{2}\right)} d x}{\int_{\mathbb{R}_{+}^{n}} \frac{u_{k_{1}}^{2}}{x_{1}^{2}} d x}=\frac{\int_{\mathbb{R}_{+}^{n}}\left|\nabla u_{k_{1}}\right|^{2} d x}{\int_{\mathbb{R}_{+}^{n}} \frac{u_{k_{1}}^{2}}{x_{1}^{2}} d x}+o(1) .
$$

To see this, let us first examine the behavior of the denominator. For $k_{1}$ large we easily compute

$$
\int_{\mathbb{R}_{+}^{n}} \frac{u_{k_{1}}^{2}}{x_{1}^{2}} d x=\int_{\mathbb{R}_{+}^{n}} x_{1}^{-1} h_{k_{1}}^{2} \phi^{2} d x>C \int_{1 / k_{1}}^{1 / 2} x_{1}^{-1} d x_{1}>C \ln k_{1}
$$

On the other hand, the terms $\sum_{i=2}^{n} \beta_{i} \int_{\mathbb{R}_{+}^{n}} \frac{u_{k_{1}}^{2}}{\left(x_{1}^{2}+\cdots+x_{i}^{2}\right)} d x$ are easily seen to be bounded as $k_{1} \rightarrow \infty$, by the Lebesgue dominated convergence theorem. From this and 2.9. we deduce 2.8.

We now estimate the gradient term in 2.8:

$$
\begin{aligned}
\int_{\mathbb{R}_{+}^{n}}\left|\nabla u_{k_{1}}\right|^{2} d x= & \frac{1}{4} \int_{\mathbb{R}_{+}^{n}} x_{1}^{-1} h_{k_{1}}^{2} \phi^{2} d x+\int_{\mathbb{R}_{+}^{n}} x_{1}\left|\nabla h_{k_{1}}\right|^{2} \phi^{2} \\
& +\int_{\mathbb{R}_{+}^{n}} x_{1} h_{k_{1}}^{2}|\nabla \phi|^{2}+\text { mixed terms. }
\end{aligned}
$$


The first integral of the right hand side behaves exactly as the denominator (cf. (2.9)), that is, it goes to infinity like $O\left(\ln k_{1}\right)$. The last integral is easily seen to be bounded as $k_{1} \rightarrow \infty$. For the middle integral we have

$$
\int_{\mathbb{R}_{+}^{n}} x_{1}\left|\nabla h_{k_{1}}\right|^{2} \phi^{2} \leq \frac{C}{\ln ^{2} k_{1}} \int_{1 / k_{1}^{2} \leq x_{1} \leq 1 / k_{1}} x_{1}^{-1} d x_{1} \leq \frac{C}{\ln k_{1}} .
$$

As a consequence of these estimates, we easily see that the mixed terms in 2.10) are of the order $o\left(\ln k_{1}\right)$ as $k_{1} \rightarrow \infty$. Hence, as $k_{1} \rightarrow \infty$,

$$
\int_{\mathbb{R}_{+}^{n}}\left|\nabla u_{k_{1}}\right|^{2} d x=\frac{1}{4} \int_{\mathbb{R}_{+}^{n}} x_{1}^{-1} h_{k_{1}}^{2} \phi^{2} d x+o\left(\ln k_{1}\right) .
$$

From 2.8, 2.9 and 2.11 we conclude that as $k_{1} \rightarrow \infty$,

$$
Q_{1}\left[u_{k_{1}}\right]=1 / 4+o(1)
$$

hence $\inf _{u \in C_{0}^{\infty}\left(\mathbb{R}_{+}^{n}\right)} Q_{1}[u] \leq 1 / 4$ and consequently $\beta_{1} \leq 1 / 4$. Therefore for a suitable nonnegative constant $\alpha_{1}$ we have $\beta_{1}=-\alpha_{1}^{2}+1 / 4$. We also set

$$
\gamma_{1}:=\alpha_{1}-1 / 2
$$

Step 2. We will next show that $\beta_{2} \leq\left(\alpha_{1}-1 / 2\right)^{2}$. To this end, setting

$$
Q_{2}[u]:=\frac{\int_{\mathbb{R}_{+}^{n}}|\nabla u|^{2} d x-\left(1 / 4-\alpha_{1}^{2}\right) \int_{\mathbb{R}_{+}^{n}} \frac{u^{2}}{x_{1}^{2}} d x-\sum_{i=3}^{n} \beta_{i} \int_{\mathbb{R}_{+}^{n}} \frac{u^{2}}{\left|\mathbf{X}_{i}\right|^{2}} d x}{\int_{\mathbb{R}_{+}^{n}} \frac{u^{2}}{\left|\mathbf{X}_{2}\right|^{2}} d x},
$$

we will prove that

$$
\inf _{u \in C_{0}^{\infty}\left(\mathbb{R}_{+}^{n}\right)} Q_{2}[u] \leq\left(\alpha_{1}-1 / 2\right)^{2} .
$$

We now consider the family of functions

$$
u_{k_{1}, k_{2}}(x):=x_{1}^{-\gamma_{1}}\left|\mathbf{X}_{2}\right|^{\alpha_{1}-1 / 2} h_{k_{1}}(x) h_{k_{2}}(x) \phi(x)=: x_{1}^{-\gamma_{1}} v_{k_{1}, k_{2}}(x) \text {. }
$$

An easy calculation shows that

$$
Q_{2}\left[u_{k_{1}, k_{2}}\right]=\frac{\int_{\mathbb{R}_{+}^{n}} x_{1}^{-2 \gamma_{1}}\left|\nabla v_{k_{1}, k_{2}}\right|^{2} d x-\sum_{i=3}^{n} \beta_{i} \int_{\mathbb{R}_{+}^{n}} x_{1}^{-2 \gamma_{1}}\left|\mathbf{X}_{i}\right|^{-2} v_{k_{1}, k_{2}}^{2} d x}{\int_{\mathbb{R}_{+}^{n}} x_{1}^{-2 \gamma_{1}}\left|\mathbf{X}_{2}\right|^{-2} v_{k_{1}, k_{2}}^{2} d x}
$$

We next use the precise form of $v_{k_{1}, k_{2}}(x)$. Concerning the denominator of $Q_{2}\left[u_{k_{1}, k_{2}}\right]$ we have

$$
\int_{\mathbb{R}_{+}^{n}} x_{1}^{-2 \gamma_{1}}\left|\mathbf{X}_{2}\right|^{-2} v_{k_{1}, k_{2}}^{2} d x=\int_{\mathbb{R}_{+}^{n}} x_{1}^{1-2 \alpha_{1}}\left(x_{1}^{2}+x_{2}^{2}\right)^{\alpha_{1}-3 / 2} h_{k_{1}}^{2} h_{k_{2}}^{2} \phi^{2} d x .
$$


Sending $k_{1}$ to infinity, using the structure of the cutoff functions and then introducing polar coordinates we get

$$
\begin{aligned}
\int_{\mathbb{R}_{+}^{n}} x_{1}^{-2 \gamma_{1}}\left|\mathbf{X}_{2}\right|^{-2} v_{\infty, k_{2}}^{2} d x & =\int_{\mathbb{R}_{+}^{n}} x_{1}^{1-2 \alpha_{1}}\left(x_{1}^{2}+x_{2}^{2}\right)^{\alpha_{1}-3 / 2} h_{k_{2}}^{2} \phi^{2} d x \\
& \geq C \int_{1 / k_{2}<x_{1}^{2}+x_{2}^{2}<1 / 2} x_{1}^{1-2 \alpha_{1}}\left(x_{1}^{2}+x_{2}^{2}\right)^{\alpha_{1}-3 / 2} d x_{1} d x_{2} \\
& \geq C \int_{0}^{\pi} \int_{1 / k_{2}}^{1 / 2} r^{-1}(\sin \theta)^{1-2 \alpha_{1}} d r d \theta \geq C \ln k_{2} .
\end{aligned}
$$

The terms in the numerator that are multiplied by the $\beta_{i}$ 's stay bounded as $k_{1}$ or $k_{2}$ go to infinity (cf. the estimates related to 2.29) in Step 3). Next,

$$
\begin{aligned}
\int_{\mathbb{R}_{+}^{n}} x_{1}^{-2 \gamma_{1}}\left|\nabla v_{k_{1}, k_{2}}\right|^{2} d x= & \left(\alpha_{1}-1 / 2\right)^{2} \int_{\mathbb{R}_{+}^{n}} x_{1}^{-2 \gamma_{1}}\left|\mathbf{X}_{2}\right|^{2 \alpha_{1}-3} h_{k_{1}}^{2} h_{k_{2}}^{2} \phi^{2} d x \\
& +\int_{\mathbb{R}_{+}^{n}} x_{1}^{-2 \gamma_{1}}\left|\mathbf{X}_{2}\right|^{2 \alpha_{1}-1}\left|\nabla\left(h_{k_{1}} h_{k_{2}}\right)\right|^{2} \phi^{2} d x \\
& +\int_{\mathbb{R}_{+}^{n}} x_{1}^{-2 \gamma_{1}}\left|\mathbf{X}_{2}\right|^{2 \alpha_{1}-1} h_{k_{1}}^{2} h_{k_{2}}^{2}|\nabla \phi|^{2} d x \\
& + \text { mixed terms. }
\end{aligned}
$$

The first integral on the right hand side above is the same as the denominator of $Q_{2}$, and therefore is finite as $k_{1} \rightarrow \infty$ and increases like $\ln k_{2}$ as $k_{2} \rightarrow \infty$ (cf. 2.16). The last integral is bounded, no matter how big the $k_{1}$ and $k_{2}$ are. Concerning the middle term we have

$$
\begin{aligned}
M\left[v_{k_{1}, k_{2}}\right]:= & \int_{\mathbb{R}_{+}^{n}} x_{1}^{-2 \gamma_{1}}\left|\mathbf{X}_{2}\right|^{2 \alpha_{1}-1}\left|\nabla\left(h_{k_{1}} h_{k_{2}}\right)\right|^{2} \phi^{2} d x \\
= & \int_{\mathbb{R}_{+}^{n}} x_{1}^{-2 \gamma_{1}}\left|\mathbf{X}_{2}\right|^{2 \alpha_{1}-1}\left|\nabla h_{k_{1}}\right|^{2} h_{k_{2}}^{2} \phi^{2} d x \\
& +\int_{\mathbb{R}_{+}^{n}} x_{1}^{-2 \gamma_{1}}\left|\mathbf{X}_{2}\right|^{2 \alpha_{1}-1} h_{k_{1}}^{2}\left|\nabla h_{k_{2}}\right|^{2} \phi^{2} d x+\text { mixed terms } \\
=: & I_{1}+I_{2}+\text { mixed terms. }
\end{aligned}
$$

Since

$$
\left|\mathbf{X}_{2}\right|^{2 \alpha_{1}-1} h_{k_{2}}^{2}=r_{2}^{2 \alpha_{1}-1} \phi_{2}\left(r_{2}\right) \leq C_{k_{2}}, \quad 0<r_{2}<1
$$

we easily get

$$
I_{1} \leq \frac{C}{\left(\ln k_{1}\right)^{2}} \int_{1 / k_{1}^{2}}^{1 / k_{1}} x_{1}^{-1-2 \alpha_{1}} d x_{1},
$$

and therefore, since $\alpha_{1} \leq 0$,

$$
I_{1} \leq \frac{C}{\ln k_{1}}, \quad k_{1} \rightarrow \infty
$$


Also, since $h_{k_{1}}^{2} \leq 1$, we similarly get (for any $k_{1}$ )

$$
I_{2} \leq \frac{C}{\left(\ln k_{2}\right)^{2}} \int_{1 / k_{2}^{2}}^{1 / k_{2}} r_{2}^{-1} d r_{2} \leq \frac{C}{\ln k_{2}}, \quad k_{2} \rightarrow \infty .
$$

From 2.18-2.20 we infer that as $k_{2} \rightarrow \infty$,

$$
M\left[v_{\infty, k_{2}}\right]=o(1) .
$$

Returning to 2.17) we see that as $k_{2} \rightarrow \infty$,

$$
\int_{\mathbb{R}_{+}^{n}} x_{1}^{-2 \gamma_{1}}\left|\nabla v_{\infty, k_{2}}\right|^{2} d x=\left(\alpha_{1}-1 / 2\right)^{2} \int_{\mathbb{R}_{+}^{n}} x_{1}^{-2 \gamma_{1}}\left|\mathbf{X}_{2}\right|^{-2} v_{\infty, k_{2}}^{2} d x+o\left(\ln k_{2}\right) .
$$

We then have as $k_{2} \rightarrow \infty$,

$$
Q_{2}\left[u_{\infty, k_{2}}\right]=\left(\alpha_{1}-1 / 2\right)^{2}+o(1) ;
$$

consequently, $\beta_{2} \leq\left(\alpha_{1}-1 / 2\right)^{2}$, and therefore $\beta_{2}=-\alpha_{2}^{2}+\left(\alpha_{1}-1 / 2\right)^{2}$ for suitable $\alpha_{2} \leq 0$. We also set

$$
\gamma_{2}=\alpha_{2}-\alpha_{1}+1 / 2
$$

Step 3. The general case. At the $(q-1)$ th step, $1 \leq q \leq n$, we have already established that

$$
\beta_{1}=-\alpha_{1}^{2}+1 / 4, \quad \beta_{m}=-\alpha_{m}^{2}+\left(\alpha_{m-1}-1 / 2\right)^{2}, \quad m=2, \ldots, q-1,
$$

for suitable nonpositive constants $a_{i}$. Also, we have defined

$$
\gamma_{1}=\alpha_{1}-1 / 2, \quad \gamma_{m}=\alpha_{m}-\alpha_{m-1}+1 / 2, \quad m=2, \ldots, q-1 .
$$

Our goal for the rest of the proof is to show that $\beta_{q} \leq\left(\alpha_{q-1}-1 / 2\right)^{2}$. To this end we consider the quotient

$$
Q_{q}[u]:=\frac{\int_{\mathbb{R}_{+}^{n}}|\nabla u|^{2} d x-\sum_{q \neq i=1}^{n} \beta_{i} \int_{\mathbb{R}_{+}^{n}} \frac{u^{2}}{\left|\mathbf{X}_{i}\right|^{2}} d x}{\int_{\mathbb{R}_{+}^{n}} \frac{u^{2}}{\left|\mathbf{X}_{q}\right|^{2}} d x} .
$$

The test function is now given by

$$
\begin{aligned}
u_{k_{1}, k_{q}}(x) & :=x_{1}^{-\gamma_{1}}\left|\mathbf{X}_{2}\right|^{-\gamma_{2}} \ldots\left|\mathbf{X}_{q-1}\right|^{-\gamma_{q-1}}\left|\mathbf{X}_{q}\right|^{\alpha_{q-1}-1 / 2} h_{k_{1}}(x) h_{k_{q}}(x) \phi(x) \\
& =: x_{1}^{-\gamma_{1}}\left|\mathbf{X}_{2}\right|^{-\gamma_{2}} \ldots\left|\mathbf{X}_{q-1}\right|^{-\gamma_{q-1}} v_{k_{q}}(x) .
\end{aligned}
$$

A straightforward calculation shows that

$$
\begin{aligned}
& Q_{q}\left[u_{k_{1}, k_{q}}\right] \\
& \qquad=\frac{\int_{\mathbb{R}_{+}^{n}} \prod_{j=1}^{q-1}\left|\mathbf{X}_{j}\right|^{-2 \gamma_{j}}\left|\nabla v_{k_{1}, k_{q}}\right|^{2} d x-\sum_{i=q+1}^{n} \beta_{i} \int_{\mathbb{R}_{+}^{n}} \prod_{j=1}^{q-1}\left|\mathbf{X}_{j}\right|^{-2 \gamma_{j}}\left|\mathbf{X}_{i}\right|^{-2} v_{k_{1}, k_{q}}^{2} d x}{\int_{\mathbb{R}_{+}^{n}} \prod_{j=1}^{q-1}\left|\mathbf{X}_{j}\right|^{-2 \gamma_{j}}\left|\mathbf{X}_{q}\right|^{-2} v_{k_{1}, k_{q}}^{2} d x} .
\end{aligned}
$$


Let us first look at the denominator,

$$
D_{q}\left[u_{k_{1}, k_{q}}\right]:=\int_{\mathbb{R}_{+}^{n}} \prod_{j=1}^{q-1}\left|\mathbf{X}_{j}\right|^{-2 \gamma_{j}}\left|\mathbf{X}_{q}\right|^{2 \alpha_{q-1}-3} h_{k_{1}}(x) h_{k_{q}}(x) \phi(x) d x .
$$

Sending $k_{1} \rightarrow \infty$, we find that $h_{k_{1}} \rightarrow 1$ and therefore

$$
D_{q}\left[u_{\infty, k_{q}}\right]=\int_{\mathbb{R}_{+}^{n}} \prod_{j=1}^{q-1}\left|\mathbf{X}_{j}\right|^{-2 \gamma_{j}}\left|\mathbf{X}_{q}\right|^{2 \alpha_{q-1}-3} h_{k_{q}}(x) \phi(x) d x .
$$

To see that this is finite we note that with $B_{R}^{+}:=\left\{x \in \mathbb{R}^{n}:|x|<R, x_{1} \geq 0\right\}$,

$$
\begin{aligned}
D_{q}\left[u_{\infty, k_{q}}\right] & \leq \int_{B_{1}^{+} \cap\left\{1 / k_{q}^{2} \leq r_{q} \leq 1 / k_{q}\right\}} \prod_{j=1}^{q-1}\left|\mathbf{X}_{j}\right|^{-2 \gamma_{j}}\left|\mathbf{X}_{q}\right|^{2 \alpha_{q-1}-3} d x \\
& \leq C \int_{\left\{1 / k_{q}^{2} \leq r_{q} \leq 1 / k_{q}\right\}} \prod_{j=1}^{q-1}\left|\mathbf{X}_{j}\right|^{-2 \gamma_{j}}\left|\mathbf{X}_{q}\right|^{2 \alpha_{q-1}-3} d x_{1} \ldots d x_{q}
\end{aligned}
$$

To estimate this, we introduce polar coordinates $\left(x_{1}, \ldots, x_{q}\right) \rightarrow\left(r_{q}, \theta_{1}, \ldots, \theta_{q-1}\right)$,

$$
\begin{aligned}
x_{1} & =r_{q} \sin \theta_{q-1} \sin \theta_{q-2} \ldots \sin \theta_{2} \sin \theta_{1} \\
x_{2} & =r_{q} \sin \theta_{q-1} \sin \theta_{q-2} \ldots \sin \theta_{2} \cos \theta_{1} \\
x_{3} & =r_{q} \sin \theta_{q-1} \sin \theta_{q-2} \ldots \cos \theta_{2} \\
& \vdots \\
x_{q} & =r_{q} \cos \theta_{q-1},
\end{aligned}
$$

where $0 \leq \theta_{1}<2 \pi$ and $0 \leq \theta_{m}<\pi$ for $m=2, \ldots, q-1$. The surface measure on the unit sphere $S^{q-1}$ then becomes

$$
C\left(\sin \theta_{q-1}\right)^{q-2}\left(\sin \theta_{q-2}\right)^{q-3} \ldots \sin \theta_{2} d \theta_{1} \ldots d \theta_{q-1} .
$$

Also, $r_{q}=\left|\mathbf{X}_{q}\right|$ and for $1 \leq m \leq q-1$,

$$
r_{m}=\left|\mathbf{X}_{m}\right|=\left(x_{1}^{2}+\cdots+x_{m}^{2}\right)^{1 / 2}=r_{q} \sin \theta_{q-1} \sin \theta_{q-2} \ldots \sin \theta_{m} .
$$

We then have

$$
\begin{aligned}
& \int_{\left\{1 / k_{q}^{2} \leq r_{q} \leq 1 / k_{q}\right\}} \prod_{j=1}^{q-1}\left|\mathbf{X}_{j}\right|^{-2 \gamma_{j}}\left|\mathbf{X}_{q}\right|^{2 \alpha_{q-1}-3} d x_{1} \ldots d x_{q} \\
& \quad=C \int_{\left\{1 / k_{q}^{2} \leq r_{q} \leq 1 / k_{q}\right\}} r_{q}^{-1} \prod_{j=1}^{q-1}\left(\sin \theta_{j}\right)^{1-2 \alpha_{j}} d \theta_{1} \ldots d \theta_{q-1} d r_{q} \leq C \ln k_{q} .
\end{aligned}
$$


On the other hand, since

$$
D_{q}\left[u_{\infty, k_{q}}\right] \geq \int_{B_{1 / 2}^{+} \cap\left\{1 / k_{q} \leq r_{q} \leq 1 / 2\right\}} \prod_{j=1}^{q-1}\left|\mathbf{X}_{j}\right|^{-2 \gamma_{j}}\left|\mathbf{X}_{q}\right|^{2 \alpha_{q-1}-3} d x,
$$

by practically the same argument we find that as $k_{q} \rightarrow \infty$,

$$
D_{q}\left[u_{\infty, k_{q}}\right] \geq C \ln k_{q}
$$

For $i=q+1, \ldots, n$, we consider the terms

$$
\begin{aligned}
\int_{\mathbb{R}_{+}^{n}} \prod_{j=1}^{q-1}\left|\mathbf{X}_{j}\right|^{-2 \gamma_{j}}\left|\mathbf{X}_{i}\right|^{-2} v_{k_{1}, k_{q}}^{2} d x=\int_{\mathbb{R}_{+}^{n}} \prod_{j=1}^{q-1}\left|\mathbf{X}_{j}\right|^{-2 \gamma_{j}}\left|\mathbf{X}_{q}\right|^{2 \alpha_{q-1}-1}\left|\mathbf{X}_{i}\right|^{-2} h_{k_{1}}^{2} h_{k_{q}}^{2} \phi(x)^{2} d x \\
\leq \int_{\mathbb{R}_{+}^{n}} \prod_{j=1}^{q-1}\left|\mathbf{X}_{j}\right|^{-2 \gamma_{j}}\left|\mathbf{X}_{q}\right|^{2 \alpha_{q-1}-1}\left|\mathbf{X}_{q+1}\right|^{-2} h_{k_{1}}^{2} h_{k_{q}}^{2} \phi(x)^{2} d x .
\end{aligned}
$$

If we let first $k_{1} \rightarrow \infty$ and then $k_{q} \rightarrow \infty$, the above integral converges to

$$
I_{q}:=\int_{\mathbb{R}_{+}^{n}} \prod_{j=1}^{q-1}\left|\mathbf{X}_{j}\right|^{-2 \gamma_{j}}\left|\mathbf{X}_{q}\right|^{2 \alpha_{q-1}-1}\left|\mathbf{X}_{q+1}\right|^{-2} \phi(x)^{2} d x .
$$

To see this is finite we introduce polar coordinates $\left(x_{1}, \ldots, x_{q+1}\right) \rightarrow\left(r_{q+1}, \theta_{1} \ldots, \theta_{q}\right)$ and use elementary estimates to get

$$
I_{q} \leq C \int_{B_{1}^{+}} \sin \theta_{q} \prod_{j=1}^{q}\left(\sin \theta_{j}\right)^{1-2 \alpha_{j}} d \theta_{1} \ldots d \theta_{q} d r_{q+1}<\infty
$$

We next consider the gradient term

$$
\begin{aligned}
& \int_{\mathbb{R}_{+}^{n}} \prod_{j=1}^{q-1}\left|\mathbf{X}_{j}\right|^{-2 \gamma_{j}}\left|\nabla v_{k_{1}, k_{q}}\right|^{2} d x \\
&=\left(\alpha_{q-1}-1 / 2\right)^{2} \int_{\mathbb{R}_{+}^{n}} \prod_{j=1}^{q-1}\left|\mathbf{X}_{j}\right|^{-2 \gamma_{j}}\left|\mathbf{X}_{q}\right|^{2 \alpha_{q-1}-3} h_{k_{1}}^{2} h_{k_{q}}^{2} \phi^{2} d x \\
& \quad+\int_{\mathbb{R}_{+}^{n}} \prod_{j=1}^{q-1}\left|\mathbf{X}_{j}\right|^{-2 \gamma_{j}}\left|\mathbf{X}_{q}\right|^{2 \alpha_{q-1}-1}\left|\nabla\left(h_{k_{1}} h_{k_{q}}\right)\right|^{2} \phi^{2} d x \\
&+\int_{\mathbb{R}_{+}^{n}} \prod_{j=1}^{q-1}\left|\mathbf{X}_{j}\right|^{-2 \gamma_{j}}\left|\mathbf{X}_{q}\right|^{2 \alpha_{q-1}-1} h_{k_{1}}^{2} h_{k_{q}}^{2}|\nabla \phi|^{2} d x+\text { mixed terms. }
\end{aligned}
$$


The first term of the right hand side is the same as the denominator. Using polar coordinates and arguments similar to the ones used in estimating the gradient term in 2.17), all other terms of 2.30) are bounded as $k_{1} \rightarrow \infty$ and $k_{q} \rightarrow \infty$. In particular, we end up with

$$
\int_{\mathbb{R}_{+}^{n}} \prod_{j=1}^{q-1}\left|\mathbf{X}_{j}\right|^{-2 \gamma_{j}}\left|\nabla v_{\infty, k_{q}}\right|^{2} d x=\left(\alpha_{q-1}-1 / 2\right)^{2} D_{q}\left[u_{\infty, k_{q}}\right]+o\left(\ln k_{q}\right), \quad k_{q} \rightarrow \infty
$$

Putting things together we obtain

$$
Q_{q}\left[u_{\infty, k_{q}}\right]=\left(\alpha_{q-1}-1 / 2\right)^{2}+o(1), \quad k_{q} \rightarrow \infty,
$$

from which it follows that $\beta_{q} \leq\left(\alpha_{q-1}-1 / 2\right)^{2}$. This completes the proof of the theorem.

The previous analysis can also lead to the following result:

Theorem 2.3. Let $\alpha_{1}, \ldots, \alpha_{k}, 1 \leq k \leq n-1$, be nonpositive constants and

$$
\beta_{1}=-\alpha_{1}^{2}+1 / 4, \quad \beta_{m}=-\alpha_{m}^{2}+\left(\alpha_{m-1}-1 / 2\right)^{2}, \quad m=2, \ldots, k
$$

Suppose that there exists a constant $\beta_{k+1}$ such that the inequality

$$
\int_{\mathbb{R}_{+}^{n}}|\nabla u|^{2} d x \geq \int_{\mathbb{R}_{+}^{n}}\left(\frac{\beta_{1}}{x_{1}^{2}}+\frac{\beta_{2}}{x_{1}^{2}+x_{2}^{2}}+\cdots+\frac{\beta_{k+1}}{x_{1}^{2}+\cdots+x_{k+1}^{2}}\right) u^{2} d x
$$

holds for any $u \in C_{0}^{\infty}\left(\mathbb{R}_{+}^{n}\right)$. Then

$$
\beta_{k+1} \leq\left(\alpha_{k}-1 / 2\right)^{2}
$$

Moreover,

$$
\inf _{u \in C_{0}^{\infty}\left(\mathbb{R}_{+}^{n}\right)} \frac{\int_{\mathbb{R}_{+}^{n}}|\nabla u|^{2} d x-\beta_{1} \int_{\mathbb{R}_{+}^{n}} \frac{|u|^{2}}{x_{1}^{2}} d x-\cdots-\beta_{k} \int_{\mathbb{R}_{+}^{n}} \frac{|u|^{2}}{x_{1}^{2}+\cdots+x_{k}^{2}} d x}{\int_{\mathbb{R}_{+}^{n}} \frac{|u|^{2}}{x_{1}^{2}+\cdots+x_{k+1}^{2}} d x}=\left(\alpha_{k}-1 / 2\right)^{2} .
$$

Proof. The proof of the first part, that is, of estimate 2.32), is contained in the proof of Theorem A(ii).

To establish (2.33), we first use 2.32 to deduce than the infimum in 2.33) is less than or equal to $\left(\alpha_{k}-1 / 2\right)^{2}$. To obtain the reverse inequality we use Theorem A(i) with $a_{k+l}=-(l-1) / 2, l=1, \ldots, n-k$. For this choice we have $\beta_{k+2}=\cdots=\beta_{n}=0$.

The following is an interesting consequence of the previous theorem. 
Corollary 2.4. For $1 \leq k \leq n$,

$$
\inf _{u \in C_{0}^{\infty}\left(\mathbb{R}_{+}^{n}\right)} \frac{\int_{\mathbb{R}_{+}^{n}}|\nabla u|^{2} d x}{\int_{\mathbb{R}_{+}^{n}} \frac{|u|^{2}}{x_{1}^{2}+\cdots+x_{k}^{2}} d x}=\frac{k^{2}}{4},
$$

and for $k \leq m<n$,

$$
\begin{aligned}
\inf _{u \in C_{0}^{\infty}\left(\mathbb{R}_{+}^{n}\right)} \frac{\int_{\mathbb{R}_{+}^{n}}|\nabla u|^{2} d x-\frac{k^{2}}{4} \int_{\mathbb{R}_{+}^{n}} \frac{|u|^{2}}{x_{1}^{2}+\cdots+x_{k}^{2}} d x-\frac{1}{4} \int_{\mathbb{R}_{+}^{n}} \frac{|u|^{2}}{x_{1}^{2}+\cdots+x_{k+1}^{2}} d x-\cdots-\frac{1}{4} \int_{\mathbb{R}_{+}^{n}} \frac{|u|^{2}}{x_{1}^{2}+\cdots+x_{m}^{2}} d x}{\int_{\mathbb{R}_{+}^{n}} \frac{|u|_{1}^{2}}{x_{1}^{2}+\cdots+x_{m+1}^{2}} d x}=\frac{1}{4} . \quad \text { (2.35) }
\end{aligned}
$$

Proof. To establish (2.34) we use (2.33) with $\alpha_{l}=-l / 2, l=1, \ldots, k-1$.

To establish 2.35] we again use (2.33) with $\alpha_{l}=-l / 2, l=1, \ldots, k-1$, and $\alpha_{l}=0$, $k \leq l \leq m$. With this choice we have $\beta_{1}=\cdots=\beta_{k-1}=0, \beta_{k}=k^{2} / 4$ and $\beta_{l}=1 / 4$, $l=k+1, \ldots, m$.

\section{Hardy-Sobolev-Maz'ya inequalities}

We begin by proving Theorem $\mathrm{C}$.

Proof of Theorem C. Our starting point is the Gagliardo-Nirenberg-Sobolev inequality

$$
C_{n} \int_{\Omega}|f|^{\frac{n}{n-1}} d x \leq\left(\int_{\Omega}|\nabla f| d x\right)^{\frac{n}{n-1}}, \quad f \in C_{0}^{\infty}(\Omega) .
$$

Let $f=\phi^{\alpha} w$, where $\alpha=\frac{2(n-1)}{n-2}$. This leads to

$$
C_{n} \int_{\Omega} \phi^{\frac{\alpha n}{n-1}}|w|^{\frac{n}{n-1}} d x \leq\left(\int_{\Omega} \alpha \phi^{\alpha-1}|\nabla \phi||w|+\phi^{\alpha}|\nabla w| d x\right)^{\frac{n}{n-1}}, \quad w \in C_{0}^{\infty}(\Omega) .
$$

We now estimate the first term in the integral according to inequality 1.5 and let $w=$ $|v|^{\theta}$. Then we get

$$
\begin{aligned}
C\left(\int_{\Omega} \phi^{\alpha n / n-1}|v|^{\theta n / n-1} d x\right)^{\frac{n-1}{n}} & \leq \int_{\Omega} \phi^{\alpha}|v|^{\theta-1}|\nabla v| d x \\
& \leq\left(\int_{\Omega} \phi^{2 \alpha-2}|v|^{2 \theta-2} d x\right)^{1 / 2}\left(\int_{\Omega} \phi^{2}|\nabla v|^{2} d x\right)^{1 / 2} .
\end{aligned}
$$

The choice

$$
\theta=\alpha=\frac{2(n-1)}{n-2}
$$


gives us the inequality

$$
C\left(\int_{\Omega} \phi^{\frac{2 n}{n-2}}|v|^{\frac{2 n}{n-2}} d x\right)^{\frac{n-2}{n}} \leq \int_{\Omega} \phi^{2}|\nabla v|^{2} d x
$$

Let $u=\phi v$. By Lemma2.1 we have

$$
\int_{\Omega}|\nabla u|^{2} d x=-\int_{\Omega} \frac{\Delta \phi}{\phi} u^{2} d x+\int_{\Omega} \phi^{2}|\nabla v|^{2} d x .
$$

We conclude the proof by combining this result with 3.2 .

Condition 1.5 might seem to be unnatural and not easily checked. However, it will be very natural and is easily verified for our choices of $\phi$.

To produce Hardy inequalities in the half-space with remainder terms also including the Sobolev term, we will need a weighted version of the Sobolev inequality.

Theorem 3.1. Let $\sigma_{1}, \ldots, \sigma_{k}$ be real numbers for some $k$ with $1 \leq k \leq n$. Set $c_{l}:=$ $\left|\sigma_{1}+\cdots+\sigma_{l}+l-1\right|$ for $1 \leq l \leq k$. Assume that

$$
c_{l} \neq 0 \quad \text { whenever } \quad \sigma_{l} \neq 0 .
$$

Then there exists a positive constant $C$ such that for any $w \in C_{0}^{\infty}\left(\mathbb{R}_{+}^{n}\right)$,

$$
\int_{\mathbb{R}_{+}^{n}} x_{1}^{\sigma_{1}}\left|\mathbf{X}_{2}\right|^{\sigma_{2}} \ldots\left|\mathbf{X}_{k}\right|^{\sigma_{k}}|\nabla w| d x \geq C\left(\int_{\mathbb{R}_{+}^{n}}\left(x_{1}^{\sigma_{1}}\left|\mathbf{X}_{2}\right|^{\sigma_{2}} \ldots\left|\mathbf{X}_{k}\right|^{\sigma_{k}}|w|\right)^{\frac{n}{n-1}} d x\right)^{\frac{n-1}{n}},
$$

and

$$
\begin{aligned}
\int_{\mathbb{R}_{+}^{n}} x_{1}^{\frac{\sigma_{1}(n-2)}{(n-1)}}\left|\mathbf{X}_{2}\right|^{\frac{\sigma_{2}(n-2)}{(n-1)}} \ldots\left|\mathbf{X}_{k}\right|^{\frac{\sigma_{k}(n-2)}{(n-1)}}|\nabla w|^{2} d x \\
\quad \geq C\left(\int_{\mathbb{R}_{+}^{n}}\left(x_{1}^{\frac{\sigma_{1}(n-2)}{2(n-1)}}\left|\mathbf{X}_{2}\right|^{\frac{\sigma_{2}(n-2)}{2(n-1)}} \ldots\left|\mathbf{X}_{k}\right|^{\frac{\sigma_{k}(n-2)}{2(n-1)}}|w|\right)^{\frac{2 n}{n-2}} d x\right)^{\frac{n-2}{n}} .
\end{aligned}
$$

Proof. For $\Omega=\mathbb{R}_{+}^{n}$ we let $u=x_{1}^{\sigma_{1}} v$ in the Sobolev inequality 3.1 to get

$$
C_{n} \int_{\mathbb{R}_{+}^{n}} x_{1}^{\frac{n \sigma_{1}}{n-1}}|v|^{\frac{n}{n-1}} d x \leq\left(\int_{\mathbb{R}_{+}^{n}}\left(\left|\sigma_{1}\right| x_{1}^{\sigma_{1}-1}|v|+x_{1}^{\sigma_{1}}|\nabla v|\right) d x\right)^{\frac{n}{n-1}}, \quad v \in C_{0}^{\infty}\left(\mathbb{R}_{+}^{n}\right) .
$$

Using the inequality

$$
\left|\int_{\mathbb{R}_{+}^{n}} \operatorname{div} \mathbf{F}\right| v|d x| \leq \int_{\mathbb{R}_{+}^{n}}|\mathbf{F}||\nabla v| d x
$$

with the vector field $\mathbf{F}=\left(x_{1}^{\sigma_{1}}, 0, \ldots, 0\right)$ one obtains

$$
\left|\sigma_{1}\right| \int_{\mathbb{R}_{+}^{n}} x_{1}^{\sigma_{1}-1}|v| d x \leq \int_{\mathbb{R}_{+}^{n}} x_{1}^{\sigma_{1}}|\nabla v| d x
$$


and hence

$$
C_{n} \int_{\mathbb{R}_{+}^{n}} x_{1}^{\frac{n \sigma_{1}}{n-1}}|v|^{\frac{n}{n-1}} d x \leq\left(\int_{\mathbb{R}_{+}^{n}} x_{1}^{\sigma_{1}}|\nabla v| d x\right)^{\frac{n}{n-1}}, \quad v \in C_{0}^{\infty}\left(\mathbb{R}_{+}^{n}\right) .
$$

Now let $v=\left|\mathbf{X}_{2}\right|^{\sigma_{2}} w=\left(x_{1}^{2}+x_{2}^{2}\right)^{\sigma_{2} / 2} w$ in the above inequality. This gives

$$
\begin{aligned}
& C_{n} \int_{\mathbb{R}_{+}^{n}} x_{1}^{\frac{n \sigma}{n-1}}\left(x_{1}^{2}+x_{2}^{2}\right)^{\frac{n \sigma_{2}}{2(n-1)}}|w|^{\frac{n}{n-1}} d x \\
& \leq\left(\int_{\mathbb{R}_{+}^{n}} x_{1}^{\sigma_{1}}\left(x_{1}^{2}+x_{2}^{2}\right)^{\sigma_{2} / 2}|\nabla w| d x+\int_{\mathbb{R}_{+}^{n}}\left|\sigma_{2}\right| x_{1}^{\sigma_{1}}\left(x_{1}^{2}+x_{2}^{2}\right)^{\sigma_{2} / 2-1 / 2}|w| d x\right)^{\frac{n}{n-1}} .
\end{aligned}
$$

Letting $\mathbf{F}=x_{1}^{\sigma_{1}}\left(x_{1}^{2}+x_{2}^{2}\right)^{\sigma_{2} / 2-1 / 2} \mathbf{X}_{2}$ in 3.5 , we get

$$
\left|\sigma_{1}+\sigma_{2}+1\right| \int_{\mathbb{R}_{+}^{n}} x_{1}^{\sigma_{1}}\left(x_{1}^{2}+x_{2}^{2}\right)^{\sigma_{2} / 2-1 / 2}|w| d x \leq \int_{\mathbb{R}_{+}^{n}} x_{1}^{\sigma_{1}}\left(x_{1}^{2}+x_{2}^{2}\right)^{\sigma_{2} / 2}|\nabla w| d x .
$$

Combining the previous two estimates we conclude that

$$
c \int_{\mathbb{R}_{+}^{n}} x_{1}^{\frac{n \sigma_{1}}{n-1}}\left(x_{1}^{2}+x_{2}^{2}\right)^{\frac{n \sigma_{2}}{2(n-1)}}|w|^{\frac{n}{n-1}} d x \leq\left(\int_{\mathbb{R}_{+}^{n}} x_{1}^{\sigma_{1}}\left(x_{1}^{2}+x_{2}^{2}\right)^{\sigma_{2} / 2}|\nabla w| d x\right)^{\frac{n}{n-1}} .
$$

Note that, in case $\sigma_{2}=0$, we have the desired result immediately and we do not have to check whether the constant $\sigma_{1}+\sigma_{2}+1$ is zero or not. We may repeat this procedure iteratively. In the $l$-th step we need the analogue of 3.6 which is

$$
\begin{aligned}
c_{l} \int_{\mathbb{R}_{+}^{n}} x_{1}^{\sigma_{1}}\left(x_{1}^{2}+x_{2}^{2}\right)^{\sigma_{2} / 2} & \ldots\left(x_{1}^{2}+\cdots+x_{l}^{2}\right)^{\left(\sigma_{l}-1\right) / 2}|w| d x \\
& \leq \int_{\mathbb{R}_{+}^{n}} x_{1}^{\sigma_{1}}\left(x_{1}^{2}+x_{2}^{2}\right)^{\sigma_{2} / 2} \ldots\left(x_{1}^{2}+\cdots+x_{l}^{2}\right)^{\sigma_{l} / 2}|\nabla w| d x
\end{aligned}
$$

for some positive constant $c_{l}$. This follows from 3.5 with

$$
\mathbf{F}=x_{1}^{\sigma_{1}}\left(x_{1}^{2}+x_{2}^{2}\right)^{\sigma_{2} / 2} \ldots\left(x_{1}^{2}+\cdots+x_{l}^{2}\right)^{\left(\sigma_{l}-1\right) / 2} \mathbf{X}_{l} .
$$

For this choice we get

$$
c_{l}=\left|\sigma_{1}+\cdots+\sigma_{l}+(l-1)\right| \text {. }
$$

So our procedure works nicely in case $c_{l} \neq 0$ for those $l$ such that $\sigma_{l} \neq 0$. This proves (3.3.

To show 3.4 we apply 3.3 to the function $w=\left.|v|\right|^{\theta}$. Trivial estimates give

$$
\begin{aligned}
C \int_{\Omega} x_{1}^{\frac{n \sigma_{1}}{n-1}} & \left(x_{1}^{2}+x_{2}^{2}\right)^{\frac{n \sigma_{2}}{2(n-1)}} \ldots\left(x_{1}^{2}+\cdots+x_{k}^{2}\right)^{\frac{n \sigma_{k}}{2(n-1)}}|v|^{\frac{n \theta}{n-1}} d x \\
& \leq\left(\theta \int_{\Omega} x_{1}^{\sigma_{1}}\left(x_{1}^{2}+x_{2}^{2}\right)^{\sigma_{2} / 2} \ldots\left(x_{1}^{2}+\cdots+x_{k}^{2}\right)^{\sigma_{k} / 2}|v|^{\theta-1}|\nabla v| d x\right)^{\frac{n}{n-1}} .
\end{aligned}
$$


We will now apply Hölder's inequality to the right hand side. We want to do it in such a way that one of the factors becomes identical to the left hand side raised to some power. Therefore we need to choose $\theta$ so that

$$
\frac{n \theta}{n-1}=2 \theta-2 \Leftrightarrow \theta=\frac{2(n-1)}{n-2} .
$$

Hölder's inequality then immediately gives the result.

We are now ready to give the proof of Theorem B:

Proof of Theorem B. For $\phi>0$ and $u=\phi v$, Lemma 2.1 gives us the inequality

$$
\int_{\mathbb{R}_{+}^{n}}|\nabla u|^{2} d x+\int_{\mathbb{R}_{+}^{n}} \frac{\Delta \phi}{\phi}|u|^{2} d x \geq \int_{\mathbb{R}_{+}^{n}} \phi^{2}|\nabla v|^{2} d x .
$$

We will choose

$$
\begin{aligned}
\phi(x) & =\left(x_{1}^{\sigma_{1}} \cdot\left(x_{1}^{2}+x_{2}^{2}\right)^{\sigma_{2} / 2} \ldots\left(x_{1}^{2}+\cdots+x_{n}^{2}\right)^{\sigma_{n} / 2}\right)^{\frac{n-2}{2(n-1)}} \\
& =\left|\mathbf{X}_{1}\right|^{-\gamma_{1}} \ldots\left|\mathbf{X}_{n}\right|^{-\gamma_{n}}
\end{aligned}
$$

where

$$
\gamma_{1}=\alpha_{1}-1 / 2, \quad \gamma_{m}=\alpha_{m}-\alpha_{m-1}+1 / 2, \quad m=2, \ldots, n,
$$

and

$$
\sigma_{m}=-\frac{2(n-1)}{n-2} \gamma_{m}, \quad m=1, \ldots, n .
$$

We now apply 3.4 of Theorem 3.1 to obtain

$$
\int_{\mathbb{R}_{+}^{n}} \phi^{2}|\nabla v|^{2} d x \geq C\left(\int_{\mathbb{R}_{+}^{n}}|\phi v|^{\frac{2 n}{n-2}} d x\right)^{\frac{n-2}{n}}
$$

provided that

$$
c_{l}:=\left|\sigma_{1}+\cdots+\sigma_{l}+l-1\right| \neq 0 \quad \text { whenever } \quad \sigma_{l} \neq 0
$$

for $1 \leq l \leq n$. Combining this with 3.7) we get

$$
\int_{\mathbb{R}_{+}^{n}}|\nabla u|^{2} d x+\int_{\mathbb{R}_{+}^{n}} \frac{\Delta \phi}{\phi}|u|^{2} d x \geq C\left(\int_{\mathbb{R}_{+}^{n}}|u|^{\frac{2 n}{n-2}} d x\right)^{\frac{n-2}{n}} .
$$

On the other hand, by Theorem A(i),

$$
-\frac{\Delta \phi}{\phi}=\frac{\beta_{1}}{x_{1}^{2}}+\frac{\beta_{2}}{x_{1}^{2}+x_{2}^{2}}+\cdots+\frac{\beta_{n}}{x_{1}^{2}+\cdots+x_{n}^{2}},
$$

and the desired inequality follows. It remains to check condition 3.9. After some elementary calculations we see that

$$
c_{l}=\frac{2(n-1)}{n-2}\left|\alpha_{l}-\frac{n-l}{2(n-1)}\right|, \quad l=1, \ldots, n .
$$


Since $\alpha_{l} \leq 0$ we clearly have $c_{l} \neq 0$ for $l=1, \ldots, n-1$. Moreover, $c_{n} \neq 0$ when $\alpha_{n}<0$. This completes the proof of (1.4).

In the rest of the proof we will show that 1.4 fails in case $\alpha_{n}=0$. To this end we will establish that

$$
\inf _{u \in C_{0}^{\infty}\left(\mathbb{R}_{+}^{n}\right)} \frac{\int_{\mathbb{R}_{+}^{n}}|\nabla u|^{2} d x-\beta_{1} \int_{\mathbb{R}_{+}^{n}} \frac{|u|^{2}}{x_{1}^{2}} d x-\cdots-\beta_{n} \int_{\mathbb{R}_{+}^{n}} \frac{|u|^{2}}{x_{1}^{2}+\cdots+x_{n}^{2}} d x}{\left(\int_{\mathbb{R}_{+}^{n}}|u|^{\frac{2 n}{n-2}} d x\right)^{\frac{n-2}{n}}}=0,
$$

where $\beta_{n}=\left(\alpha_{n-1}-1 / 2\right)^{2}$. Let

$$
u(x)=x_{1}^{-\gamma_{1}}\left|\mathbf{X}_{2}\right|^{-\gamma_{2}} \ldots\left|\mathbf{X}_{n-1}\right|^{-\gamma_{n-1}} v(x) .
$$

A straightforward calculation, quite similar to the one leading to 2.15, shows that the infimum in 3.10 is the same as

$$
\inf _{v \in C_{0}^{\infty}\left(\mathbb{R}_{+}^{n}\right)} \frac{\int_{\mathbb{R}_{+}^{n}} \prod_{j=1}^{n-1}\left|\mathbf{X}_{j}\right|^{-2 \gamma_{j}}|\nabla v|^{2} d x-\beta_{n} \int_{\mathbb{R}_{+}^{n}} \prod_{j=1}^{n-1}\left|\mathbf{X}_{j}\right|^{-2 \gamma_{j}}\left|\mathbf{X}_{n}\right|^{-2} v^{2} d x}{\left(\int_{\mathbb{R}_{+}^{n}}\left(\prod_{j=1}^{n-1}\left|\mathbf{X}_{j}\right|^{-\gamma_{j}}\right)^{\frac{2 n}{n-2}}|v|^{\frac{2 n}{n-2}} d x\right)^{\frac{n-2}{n}}} .
$$

We now choose the test functions

$$
v_{k_{1}, \varepsilon}=\left|\mathbf{X}_{n}\right|^{-\gamma_{n}+\varepsilon} h_{k_{1}}(x) \phi(x), \quad \varepsilon>0,
$$

where $h_{k_{1}}(x)$ and $\phi(x)$ are as in the first step of the proof of Theorem A(ii). For this choice, after straightforward calculations, quite similar to the ones used in the proof of Theorem A(ii), we obtain the following estimate for the numerator $N$ in (3.11):

$$
\begin{aligned}
N\left[v_{\infty, \varepsilon}\right]= & \left(\left(\alpha_{n-1}-1 / 2+\varepsilon\right)^{2}-\left(\alpha_{n-1}-1 / 2\right)^{2}\right) \\
& \times \int_{\mathbb{R}_{+}^{n}} \prod_{j=1}^{n-1}\left|\mathbf{X}_{j}\right|^{-2 \gamma_{j}}\left|\mathbf{X}_{n}\right|^{-2 \gamma_{n}+2+\varepsilon} \phi(x)^{2} d x+O_{\varepsilon}(1) \\
= & C \varepsilon \int_{\mathbb{R}_{+}^{n}} r^{-1+2 \varepsilon} \prod_{j=1}^{n}\left(\sin \theta_{j}\right)^{1-2 \alpha_{j}} \phi(r)^{2} d \theta_{1} \ldots d \theta_{n-1} d r+O_{\varepsilon}(1) \\
= & C \varepsilon \int_{0}^{1} r^{-1+\varepsilon} d r+O_{\varepsilon}(1) .
\end{aligned}
$$

In the above calculations we have taken the limit $k_{1} \rightarrow \infty$ and we have used polar coordinates $\left(x_{1}, \ldots, x_{n}\right) \rightarrow\left(\theta_{1}, \ldots, \theta_{n-1}, r\right)$. We then conclude that

$$
N\left[v_{\infty, \varepsilon}\right]<C \quad \text { as } \varepsilon \rightarrow 0 .
$$

Similar calculations for the denominator $D$ in 3.11 reveal that

$$
\begin{aligned}
D\left[v_{\infty, \varepsilon}\right] & =C\left(\int_{\mathbb{R}_{+}^{n}} r^{-1+\frac{2 \varepsilon n}{n-2}} \prod_{j=1}^{n-1}\left(\sin \theta_{j}\right)^{\frac{n-j}{n-2}-\frac{2 n \alpha_{j}}{n-2}-1} \phi^{\frac{2 n}{n-2}} d \theta_{1} \ldots d \theta_{n-1} d r\right)^{\frac{n-2}{n}} \\
& \geq C\left(\int_{0}^{1 / 2} r^{-1+\frac{2 \varepsilon n}{n-2}} d r\right)^{\frac{n-2}{n}}=C \varepsilon^{-\frac{n-2}{n}}
\end{aligned}
$$


We then have

$$
\frac{N\left[v_{\infty, \varepsilon}\right]}{D\left[v_{\infty, \varepsilon}\right]} \rightarrow 0 \quad \text { as } \varepsilon \rightarrow 0,
$$

and therefore the infimum in (3.11) or 3.10) is equal to zero. This completes the proof of the theorem.

Here is a consequence of Theorem B.

Corollary 3.2. Let $1 \leq k<n$. For any $\beta_{n}<1 / 4$, there exists a positive constant $C$ such that for all $u \in C_{0}^{\infty}\left(\mathbb{R}_{+}^{n}\right)$,

$$
\begin{aligned}
\int_{\mathbb{R}_{+}^{n}}|\nabla u|^{2} d x \geq & \int_{\mathbb{R}_{+}^{n}}\left(\frac{k^{2}}{4} \frac{1}{x_{1}^{2}+\cdots+x_{k}^{2}}+\frac{1}{4} \frac{1}{x_{1}^{2}+\cdots+x_{k+1}^{2}}+\cdots\right. \\
& \left.+\frac{1}{4} \frac{1}{x_{1}^{2}+\cdots+x_{n-1}^{2}}+\frac{\beta_{n}}{x_{1}^{2}+\cdots+x_{n}^{2}}\right)|u|^{2} d x+C\left(\int_{\mathbb{R}_{+}^{n}}|u|^{2^{*}} d x\right)^{2 / 2^{*}} .
\end{aligned}
$$

If $\beta_{n}=1 / 4$ the previous inequality fails.

In case $k=n$ for any $\beta_{n}<n^{2} / 4$, there exists a positive constant $C$ such that for all $u \in C_{0}^{\infty}\left(\mathbb{R}_{+}^{n}\right)$,

$$
\int_{\mathbb{R}_{+}^{n}}|\nabla u|^{2} d x \geq \beta_{n} \int_{\mathbb{R}_{+}^{n}} \frac{|u|^{2}}{x_{1}^{2}+\cdots+x_{n}^{2}} d x+C\left(\int_{\mathbb{R}_{+}^{n}}|u|^{2^{*}} d x\right)^{2 / 2^{*}} .
$$

The above inequality fails for $\beta_{n}=n^{2} / 4$.

Proof. In Theorem B we make the following choices: In the case $k=1$ we choose $\alpha_{1}=\cdots=\alpha_{n-1}=0$. In this case $\beta_{k}=1 / 4, k=1, \ldots, n-1$. The condition $\alpha_{n}<0$ is equivalent to $\beta_{n}<1 / 4$.

In the case $1<k \leq n-1$ we choose $\alpha_{m}=-m / 2$ when $m=1, \ldots, k-1$, and $\alpha_{m}=0$ when $m=k, \ldots, n-1$. Finally, in case $k=n$ we choose $\alpha_{m}=-m / 2$ for $m=1, \ldots, n-1$.

\section{Further generalizations}

The techniques used in the previous sections can be generalized to other situations as well. For example, consider the subset of $\mathbb{R}^{n}$ where $x_{1}, \ldots, x_{k}>0$. We denote this domain by $\mathbb{R}_{k_{+}}^{n}$. Then we can easily prove the Hardy-Sobolev inequality

Theorem 4.1. There exists a positive constant $C$ such that for any $u \in C_{0}^{\infty}\left(\mathbb{R}_{k_{+}}^{n}\right)$

$$
\int_{\mathbb{R}_{k_{+}}^{n}}|\nabla u|^{2} d x \geq \frac{1}{4} \int_{\mathbb{R}_{k_{+}}^{n}}\left(\frac{1}{x_{1}^{2}}+\cdots+\frac{1}{x_{k}^{2}}\right)|u|^{2} d x+C\left(\int_{\mathbb{R}_{k_{+}}^{n}}|u|^{2^{*}} d x\right)^{2 / 2^{*}} .
$$


Proof. Let $\phi=\sqrt{x_{1} \ldots x_{k}}$. For $u=\phi w$ we calculate

$$
\begin{aligned}
\int_{\mathbb{R}_{k_{+}}^{n}}|\nabla u|^{2} d x= & \int_{\mathbb{R}_{k_{+}}^{n}}\left|\sqrt{x_{1} \ldots x_{k}} \cdot \nabla w+\frac{1}{2} \sqrt{x_{1} \ldots x_{k}}\left(\frac{1}{x_{1}}, \ldots, \frac{1}{x_{k}}\right) w\right|^{2} d x \\
= & \int_{\mathbb{R}_{k_{+}}^{n}} x_{1} \ldots x_{k}|\nabla w|^{2} d x+\frac{1}{4} \int_{\mathbb{R}_{k_{+}}^{n}} x_{1} \ldots x_{k}\left(\frac{1}{x_{1}^{2}}+\cdots+\frac{1}{x_{k}^{2}}\right)|w|^{2} d x \\
& +\frac{1}{2} \int_{\mathbb{R}_{k_{+}}^{n}} x_{1} \ldots x_{k}\left(\frac{1}{x_{1}}, \ldots, \frac{1}{x_{k}}\right) \nabla w^{2} d x .
\end{aligned}
$$

By partial integration, we see that the last term is zero. If the second term is expressed in terms of $u$, we see that it is equal to the Hardy term

$$
\frac{1}{4} \int_{\mathbb{R}_{k_{+}}^{n}}\left(\frac{1}{x_{1}^{2}}+\cdots+\frac{1}{x_{k}^{2}}\right)|u|^{2} d x .
$$

By Theorem $C$, the first term may be estimated from below by the Sobolev term provided that we can prove the following $L^{1}$ Hardy inequality:

$$
C \int_{\mathbb{R}_{k_{+}}^{n}}\left(x_{1} \ldots x_{k}\right)^{\frac{n-1}{n-2}}\left(\frac{1}{x_{1}^{2}}+\cdots+\frac{1}{x_{k}^{2}}\right)^{1 / 2}|v| d x \leq \int_{\mathbb{R}_{k_{+}}^{n}}\left(x_{1} \ldots x_{k}\right)^{\frac{n-1}{n-2}}|\nabla v| d x .
$$

To do this we work as in the previous section, using the inequality

$$
\left|\int_{\mathbb{R}_{k_{+}}^{n}} \operatorname{div} \mathbf{F}\right| v|d x| \leq \int_{\mathbb{R}_{k_{+}}^{n}}|| \mathbf{F}|\nabla v| d x
$$

with the proper choice of vector field, which turns out to be

$$
\mathbf{F}=\left(x_{1} \ldots x_{k}\right)^{\tau}\left(\frac{1}{x_{1}^{2}}+\cdots+\frac{1}{x_{k}^{2}}\right)^{\beta}\left(\frac{1}{x_{1}}, \ldots, \frac{1}{x_{k}}\right)
$$

where

$$
\tau=\frac{n-1}{n-2} \quad \text { and } \quad \beta=-\frac{1}{2} .
$$

We immediately see that $|\mathbf{F}|=\phi^{2 \tau}=\left(x_{1} \ldots x_{k}\right)^{\frac{n-1}{n-2}}$. Also,

$$
\begin{aligned}
\operatorname{div} \mathbf{F}= & -\left(x_{1} \ldots x_{k}\right)^{\tau}\left(\frac{1}{x_{1}^{2}}+\cdots+\frac{1}{x_{k}^{2}}\right)^{\beta+1} \\
& +\tau\left(x_{1} \ldots x_{k}\right)^{\tau}\left(\frac{1}{x_{1}^{2}}+\cdots+\frac{1}{x_{k}^{2}}\right)^{\beta+1} \\
& -2 \beta\left(\frac{1}{x_{1}^{4}}+\cdots+\frac{1}{x_{k}^{4}}\right) \cdot\left(x_{1} \ldots x_{k}\right)^{\tau}\left(\frac{1}{x_{1}^{2}}+\cdots+\frac{1}{x_{k}^{2}}\right)^{\beta-1} .
\end{aligned}
$$

Since $\tau-1>0$ and the last term is positive, we get the result. 
Acknowledgments. This work was largely done whilst JT was visiting the University of Crete and FORTH in Heraklion, supported by a postdoctoral fellowship through the RTN European network Fronts-Singularities, HPRN-CT-2002-00274. SF and AT acknowledge partial support by the same program.

\section{References}

[1] Ancona, A.: On strong barriers and an inequality of Hardy for domains in $\mathbb{R}^{n}$. J. London Math. Soc. 34, 274-290 (1986) Zbl 0629.31002 MR 0856511

[2] Barbatis, G., Filippas, S., Tertikas, A.: A unified approach to improved $L^{p}$ Hardy inequalities with best constants. Trans. Amer. Math. Soc. 356, 2169-2196 (2004) Zbl 1129.26019 MR 2048514

[3] Benguria, R. D., Frank, R. L., Loss, M.: The sharp constant in the Hardy-Sobolev-Maz'ya inequality in the three dimensional upper half space. Math. Res. Lett. 15, 613-622 (2008) Zbl pre05492427 MR 2424899

[4] Brezis, H., Marcus, M.: Hardy's inequalities revisited. Ann. Scuola Norm. Sup. Pisa Cl. Sci. 25, 217-237 (1997) Zbl 1011.46027 MR 1655516

[5] Davies, E. B.: The Hardy constant. Quart. J. Math. 46, 417-431 (1995) Zbl 0857.26005 MR 1366614

[6] Dávila, J., Dupaigne, L.: Hardy-type inequalities. J. Eur. Math. Soc. 6, 335-365 (2004) Zbl 1083.26012 MR 2060479

[7] Filippas, S., Maz'ya, V., Tertikas, A.: On a question of Brezis and Marcus. Calc. Var. Partial Differential Equations 25, 491-501 (2006) Zbl 1121.26014 MR 2214621

[8] Filippas, S., Maz'ya, V., Tertikas, A.: Critical Hardy-Sobolev inequalities. J. Math. Pures Appl. 87, 37-56 (2007) Zbl pre05134242 MR 2297247

[9] Hoffmann-Ostenhof, M., Hoffmann-Ostenhof, T., Laptev, A.: A geometrical version of Hardy's inequality. J. Funct. Anal. 189, 539-548 (2002) Zbl 1012.26011 MR 1892180

[10] Maz'ya, V.: Sobolev Spaces. Springer, Berlin (1985). Zbl 0692.46023 MR 0817985

[11] Tertikas, A., Tintarev, K.: On existence of minimizers for the Hardy-Sobolev-Maz'ya inequality. Ann. Mat. Pura Appl. 186, 645-662 (2007) Zbl pre05214380 MR 2317783

[12] Tidblom, J.: A geometrical version of Hardy's inequality for $\stackrel{\circ}{W}^{1, p}(\Omega)$. Proc. Amer. Math. Soc. 132, 2265-2271 (2004) Zbl 1062.26010 MR 2052402

[13] Tidblom, J.: A Hardy inequality in the half-space. J. Funct. Anal. 221, 482-495 (2005) Zbl 1077.26010 MR 2124873 